\section{Mechanical testing of lid speculae and relationship to postoperative ptosis}

\begin{abstract}
Aims Postoperative lid malpositions are known complications of routine intraocular surgery and were previously attributed to the use of a bridle suture or the myotoxic effect of retrobulbar or peribulbar anaesthetics. However, lid malpositions are still seen under topical anaesthesia. Recent studies have implicated the lid speculum as a factor. Patients with narrower vertical palpebral apertures have been shown to develop postoperative ptosis more frequently, but the reason is unknown. This is the first study to determine the forces exerted by lid speculae over a range of palpebral apertures. Methods Mechanical testing was undertaken using a Bose 3200 materials testing machine. Tests were undertaken on four disposable and four reusable speculae. The force used to compress each speculum was compared over a range of displacements. A two-sample $t$-test was used to compare the stiffness of the two types of speculum.

Results The stiffness of the reusable speculum was significantly greater than the disposable speculum $(P=0.002)$. The stiffness of each speculum was greatest at the range of displacement corresponding to the narrower palpebral apertures.

Conclusions Different speculae exert significantly different forces on patients' eyelids during surgery. The patients who experience the greatest compression from the speculae are those with the smallest palpebral apertures. This may explain why these patients are more likely to develop postoperative lid malpositions. Eye (2013) 27, 1098-1101; doi:10.1038/eye.2013.133; published online 21 June 2013
\end{abstract}

Keywords: blepharoptosis; ectropion; epiphora; postoperative complications; cataract extraction; speculum
NJ Crosby ${ }^{1}$, D Shepherd ${ }^{2}$ and A Murray ${ }^{1}$

\section{Introduction}

Postoperative ptosis, ectropion, and epiphora are frequently overlooked complications of routine intraocular surgery. Estimates of the incidence of postoperative ptosis vary from $7.3 \%$ to $21 \%$ after cataract surgery, ${ }^{1-3} 10 \%$ after radial keratotomy, ${ }^{4}$ and $10.7 \%$ after trabeculectomy. ${ }^{5}$ The incidence of entropion or ectropion after intraocular surgery is not known, although Hosal et al ${ }^{1}$ demonstrated an incidence of punctal ectropion of $4 \%$ after cataract surgery.

Various factors have been suggested as a cause of postoperative lid malpositions, including lid oedema, use of a bridle suture, the myotoxicity of peribulbar or retrobulbar anaesthetics, and the lid speculum. Recent studies have shown the lid speculum to be a significant factor in postoperative ptosis. $2,4,6$

Patients with postoperative ptosis have been found to have disinsertion of the levator palpebrae superioris (LPS) aponeurosis. ${ }^{7-9}$ It has been postulated that contraction of the orbicularis oculi against the speculum may cause dehiscence of the LPS aponeurosis ${ }^{4}$ or that the speculum may compress the lid against the orbital rim causing inflammation and oedema, which may result in weakening of the aponeurosis. $^{2,7}$ By a similar mechanism, postoperative entropion may be caused by dehiscence of the capsulopalpebral fascia. ${ }^{10}$

From our own observations and on the basis of previous studies of postoperative lid malpositions, we developed the following hypothesis: that stiffer speculae are more likely to cause dehiscence of the LPS aponeurosis and lid malpositions, and that patients with smaller palpebral apertures and tight lids would be more vulnerable to speculum-induced damage. As the first step to test this theory, we measured the stiffness of different speculae over a range of palpebral apertures. We tested different 
speculae to determine whether there was a difference in the stiffness of different types.

\section{Materials and methods}

Mechanical testing was undertaken using a Bose 3200 materials testing machine (Bose Corporation,

ElectroForce Systems Group, Eden Prairie, MN, USA). Tests were undertaken on four disposable Barraquer stainless steel speculae (Malosa Medical, Elland, UK) and four reusable Kratz Barraquer titanium speculae (Altomed, Boldon, UK). Each speculum was clamped to the base of the testing machine. A spherical indenter was attached to the actuator of the testing machine and this was used to compress the speculum at a rate of $0.1 \mathrm{~mm} / \mathrm{s}$ (Figure 1). Force and displacement were recorded through the testing. Graphs of force against displacement were plotted and a line in the form $F=\mathrm{kx}$ was fitted to each set of data, were $F$ is the force, $x$ is the displacement, and $k$ is the gradient of the line, which is the stiffness of the speculum. All statistical analysis was performed using either Excel 2003 (Microsoft, Reading, UK) or Minitab 15 (Minitab Inc., State College, PA, USA).

\section{Results}

Figure 2 shows a plot of force against displacement for a disposable and a reusable speculum. It can be seen that the gradient of the line (stiffness) is greater for the reusable speculum compared with the disposable speculum. The stiffness values for the speculae are shown in Table 1. The stiffness values of the reusable speculae were in the range $0.1325-0.1375 \mathrm{~N} / \mathrm{mm}$, with a mean and SD of 0.1348 and $0.0026 \mathrm{~N} / \mathrm{mm}$, respectively. For the disposable speculae the mean stiffness was $0.0966 \mathrm{~N} / \mathrm{mm}$ with an SD of $0.0070 \mathrm{~N} / \mathrm{mm}$. The range of values was $0.0871-0.1037 \mathrm{~N} / \mathrm{mm}$. A two-sample $t$-test

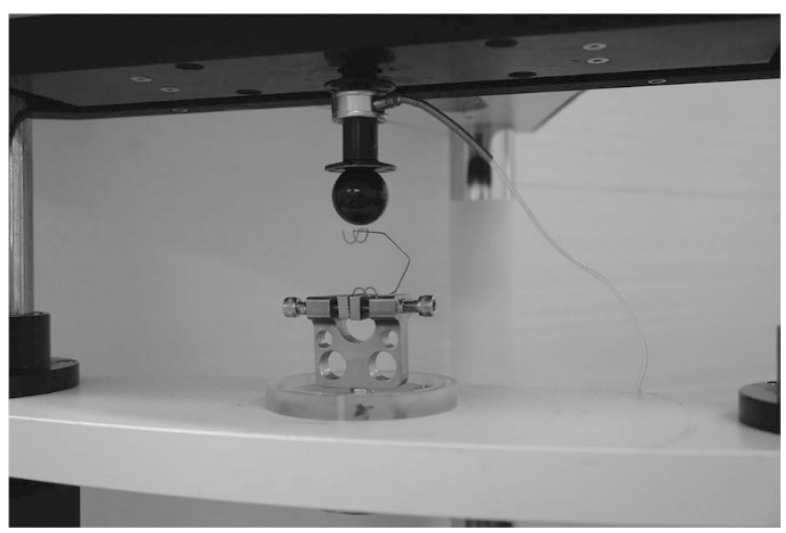

Figure 1 Speculum testing. showed that the stiffness of the reusable speculum was significantly greater than the disposable speculum $(P=0.002)$.

\section{Conclusion}

Ptosis, entropion, ectropion, and epiphora are known complications of routine intraocular surgery, although patients are rarely warned that they may occur. Ptosis has been reported in as many as $44.4 \%$ of patients undergoing routine cataract surgery at 6 weeks, ${ }^{6}$ although for the majority of patients this resolves without treatment. ${ }^{11}$ Causes of temporary ptosis are thought to include eyelid oedema, indirect infiltration of the LPS by retrobulbar or peribulbar anaesthesia, and ocular surface disturbance. ${ }^{11}$

Permanent postoperative ptosis is widely thought to be due to dehiscence of the LPS aponeurosis: the clinical features of the ptosis include good levator function and a

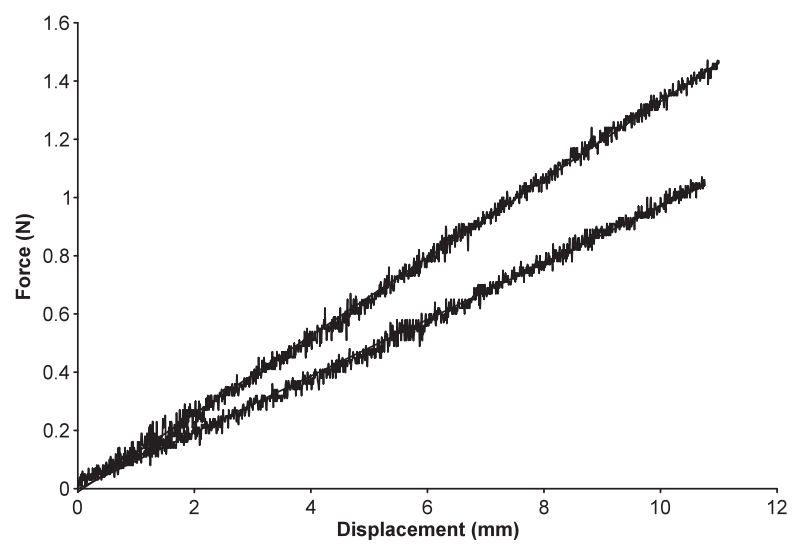

Figure 2 Force against displacement for a disposable speculum (sample 3) and a reusable speculum (sample 3). The upper curve is for the reusable speculum with a curve fit of $F=0.1325 x$. The lower curve is for the disposable speculum with $F=0.0968 \mathrm{x}$.

Table 1 Values of the constant $\mathrm{A}$ for the line $F=\mathrm{Ax}$

\begin{tabular}{lcc}
\hline Speculum & Stiffness $(\mathrm{N} / \mathrm{mm})$ & $\mathrm{R}^{2}$ \\
\hline Disposable 1 & 0.0871 & 0.992 \\
Disposable 2 & 0.1037 & 0.992 \\
Disposable 3 & 0.0968 & 0.996 \\
Disposable 4 & 0.0988 & 0.996 \\
Reusable 1 & 0.1375 & 0.987 \\
Reusable 2 & 0.1327 & 0.997 \\
Reusable 3 & 0.1325 & 0.997 \\
Reusable 4 & 0.1364 & 0.997
\end{tabular}

$R^{2}$ is a squared correlation coefficient and shows how well the line fits the data points. 
high lid crease. Furthermore, Paris and Quickert ${ }^{7}$ observed disinsertion of the LPS aponeurosis from the epitarsus during post-cataract ptosis repair. Others have since repeated this observation..$^{8,9}$ Deady et al ${ }^{12}$ observed that patients who developed a post-cataract ptosis were more likely to have a pre-existing ptosis on the contralateral (unoperated) side. They postulated that these patients had weakened LPS aponeuroses, which made them vulnerable to postoperative ptosis.

Various factors have been investigated as a cause of the LPS aponeurosis dehiscence including lid oedema, the use of a bridle suture, the speculum, or a combination of these factors. Paris and Quickert ${ }^{7}$ speculated that prolonged eyelid oedema caused dehiscence of the LPS aponeurosis. However, subsequent studies have found no association between the amount of lid oedema and subsequent postoperative ptosis. ${ }^{3,6}$ Singh et $a l^{6}$ found the rate of postoperative ptosis to be almost doubled when a speculum and a superior rectus bridle suture were used together, compared with a bridle suture alone. They concluded that the speculum pulls the LPS aponeurosis superiorly whereas the bridle suture pulls the superior rectus downward. As the superior rectus and LPS are strongly attached, this was thought to result in dehiscence of the relatively weak LPS aponeurosis. However, Patel et $a l^{2}$ found the rate of postoperative ptosis to be the same whether a bridle suture was used or not. They concluded that other factors, including the speculum, were responsible.

Linberg et al $^{4}$ reported ptosis after radial keratotomy and concluded that the speculum was responsible. They used a rigid speculum opened widely, under topical anaesthesia with no bridle sutures or lid block. They speculated that trauma to the lid and thus to the LPS aponeurosis results from contraction of the orbicularis oculi against the rigid speculum.

Lower lid malpositions after intraocular surgery have been less widely studied, and the incidence of entropion or ectropion after intraocular surgery is not known. Hosal et al ${ }^{1}$ studied 124 patients undergoing extracapsular cataract extraction with retrobulbar anaesthesia and a Van Lint facial nerve block, a flexible wire speculum, and a superior rectus bridle suture. In all, $4 \%$ of these patients developed punctal ectropion. It has been postulated that postoperative entropion or ectropion may be caused by dehiscence of the capsulopalpebral fascia in a similar manner to that of the LPS aponeurosis in postoperative ptosis. ${ }^{10}$ The cause of this putative dehiscence is also not known.

Until now, no one has measured the stiffness of lid speculae. Our results show a significant difference in the stiffness between two types of lid speculae widely used in practice, with the reusable speculae having a greater stiffness. This means that when the reusable speculum is used it will exert a greater force on the lid, for the same given displacement. We hypothesize that the use of stiffer speculae are associated with an increased incidence of postoperative lid malpositions.

Furthermore, we would anticipate that patients with smaller palpebral apertures and tight eyelids are more vulnerable to these complications with the stiffer speculae, as a result of the greater force exerted.

This hypothesis is supported by previous studies. Linberg et al ${ }^{4}$ thought that a rigid speculum was the cause of postoperative ptosis in their patients. Hosal et al ${ }^{1}$ found that patients with smaller palpebral apertures were more likely to develop ptosis. Lower incidences of postoperative ptosis have been reported after general anaesthesia, compared with local anaesthesia. ${ }^{12,13}$ One reason may be that under general anaesthesia muscle relaxants prevent patients from squeezing against a rigid speculum, whereas they are not prevented from doing this under local anaesthesia.

We suggest that the more rigid speculae are avoided in patients with small palpebral apertures and vulnerable LPS aponeuroses. We are now planning a clinical study to look into the in vivo variation in forces exerted on eyelids to determine whether this correlates with subsequent lid malpositions.

\section{Summary}

What was known before

- Postoperative lid malpositions are known complications of routine intraocular surgery, even under topical anaesthesia. Recent studies have implicated the lid speculum as a factor. Patients with narrower vertical palpebral apertures have been shown to develop postoperative ptosis more frequently, but the reason is unknown.

What this study adds

- Different speculae exert significantly different forces on patients' eyelids during surgery. This study shows that the patients who will experience the greatest compression from the speculae are those with the smallest palpebral apertures. This may explain why these patients are more likely to develop postoperative lid malpositions.

\section{Conflict of interest}

The authors declare no conflict of interest.

\section{Acknowledgements}

We thank Arthritis Research UK for funding the equipment used in this study (Grant number H0671). 


\section{References}

1 Hosal BM, Tekeli O, Gürsel E. Eyelid malpositions after cataract surgery. Eur J Ophthalmol 1998; 8: 12-15.

2 Patel JI, Blount M, Jones C. Surgical blepharoptosis - the bridle suture factor? Eye 2002; 16: 535-537

3 Kaplan LJ, Jaffe NS, Clayman HM. Ptosis and cataract surgery. A multivariant computer analysis of a prospective study. Ophthalmology 1985; 92: 237-242.

4 Linberg JV, McDonald MB, Safir A, Googe JM. Ptosis following radial keratotomy: performed using a rigid eyelid speculum. Ophthalmology 1986; 93: 1509-1512.

5 Song MS, Shin DH, Spoor TC. Incidence of ptosis following trabeculectomy: a comparative study. Korean J Ophthalmol 1996; 10: 97-103.

6 Singh SK, Sekhar GC, Gupta S. Etiology of ptosis after cataract surgery. J Cataract Refract Surg 1997; 23: 1409-1413.
7 Paris GL, Quickert MH. Disinsertion of the aponeurosis of the levator palpebrae superioris muscle after cataract extraction. Am J Ophthalmol 1976; 81: 337-340.

8 Baggio E, Ruban JM. Postoperative ptosis: etiopathogenesis, clinical analysis, and therapeutic management. Apropos of a series of 43 cases. J Fr Ophtalmol 1998; 21: 361-373.

9 Parsa FD, Wolff DR, Parsa NN, Elahi AE. Upper eyelid ptosis repair after cataract extraction and the importance of Hering's test. Plast Reconstr Surg 2001; 108: 1527-1536; discussion 1537-1538.

10 Hurwitz JJ, Smith D, Corin SM. Association of entropion with cataract surgery. Ophthal Plast Reconstr Surg 1990; 6: 25-27.

11 Bernardino CR, Rubin PAD. Ptosis after cataract surgery. Semin Ophthalmol 2002; 17: 144-148.

12 Deady JP, Price NJ, Sutton GA. Ptosis following cataract and trabeculectomy surgery. Br J Ophthalmol 1989; 73: 283-285.

13 Alpar JJ. Acquired ptosis following cataract and glaucoma surgery. Glaucoma 1982; 4: 66-68. 